\title{
Transforming the Traditional Drug Development Model
}

\author{
Arkesh Mehta ${ }^{1,2 *}$ and Jenny Smith ${ }^{1}$ \\ ${ }^{1}$ OncoBindi Therapeutics, USA \\ ${ }^{2}$ Germinate 360, USA
}

Submission: March 29, 2018; Published: April 09, 2018

*Corresponding author: Arkesh Mehta, OncoBindi Therapeutics, 30 N Gould Suite 4000 Sheridan WY, 82801, USA, Tel: 1-240-422-2118;

Email: amehta@oncobindi.com

\section{Introduction}

For more than a decade, the pharmaceutical industry has been facing mounting public, government, and internal pressure to contain development costs, lower drug prices, and ramp up pipelines faster. At the same time, the cost, complexity and globalization of drug development continues to escalate. These challenges plus financial, political and scientific developments are driving a transformation in the traditional biopharmaceutical business model.

Bringing a drug to market is a slow, expensive, and risky business. It takes about 10-20 years to develop a compound, and the failure rate is nearly 95\% [1]. A report by the Tufts Center for the Study of Drug Development pegs the cost at $\$ 2.6$ billion [2]. Getting a product to market in some cases can potentially cost more than $\$ 5$ billion, according to an analysis by Forbes [3]. Biopharmaceutical companies, governments, society and patients can no longer bear to conduct business of improving healthcare for all as usual.

Clearly the traditional "bench-to-bedside" drug development model is unsustainable under current market conditions. As drug sponsors seek new ways to meet the challenges of today's stringent business and industry climate, they are reinventing themselves, adopting new strategies to build product pipelines more efficiently. The pressure to develop personalized precision therapeutics by increasingly active patient groups demanding clinical outcome focused drug development increases the complexity of developing sustainable product portfolios in a cost efficient fashion.

This article discusses emerging strategies for making drug development more efficient while reducing costs and risk, such as completely transforming the standard processes in a traditional drug development model. This article describes a transformative approach to drug design and development. The proposed unified architecture of the drug development is simple, globally scalable, and globally collaborative. The transformative platform for development is independent of drug properties or the disease domain. The technology platform meets the critical objectives of being clinical outcome focus, patient-centricity, operational efficiency, sustainability and being future-proof.

\section{New Strategies, New Business Model}

Over the last few decades the biopharmaceutical drug development has seen some incremental innovations that have not made a significant impact on the operational efficiency of the drug development. However, these approaches along with big data centric approaches will only bring in incremental or disruptive changes. What biopharmaceutical companies need is transformative innovation to develop next generation of clinical outcome focused precision therapeutics. In fact, the cost of drug development has gone significantly higher with unreasonable timelines. At the same time patient groups and reimbursement providers are demanding better clinical outcomes and cost efficiencies. It provides a tremendous opportunity for a unified drug development platform for next generation of clinical outcome focused-precision therapeutics.

To become the nextgeneration of innovative biopharmaceutical companies with some sustainable and future-proof portfolios with an operationally efficient infrastructure a comprehensive strategy is a must. The stake holders demand patient centric precision therapeutics development that meet some or all of the following criteria:

Some of the desired properties for a clinical outcomefocused, precision therapeutic drug:

a) Simple, Unified or Integrated, Globally scalable, Collaborative, Sustainable,

b) Future-proof, Cost effective, Time efficient, Regulatory agency friendly, Independent of drug properties, Independent of disease domain, modular

c) Coupled with these objectives, biopharmaceutical companies need to transform the traditional development pathway to reduce the high risk of failure, focusing on the 
economics of development and market acceptance at the outset.

\section{Establishing the Operationally Efficient Drug Development Technology Infrastructure}

Leveraging innovative drug development technologies can facilitate determining a drug's clinical efficacy and result in patient benefit. Advanced technology can combine historical and clinical data related to previous trials of similar agents, and help predict the likely profitability of a drug based on the historical analysis of similar agents and size of the potential market.

\section{Innovative Strategies for Pipeline Growth that is Sustainable and Future-Proof}

Dramatic changes are on the drug development horizon as biopharmaceutical sponsors struggle to keep pace with the demands of today's stringent industry environment. To grow revenues and build pipelines, sponsors must bolster R \& D efficiency and innovation. Current drug development models are inadequate to meet these demands. Some larger sponsors are beginning to apply new strategies and business models, leveraging innovative technologies to achieve significant timeand cost-savings, improve decision-making and gain tremendous value in drug development. The increased patient awareness demands precision therapeutics that is free of adverse drug effects. The increased investor activism dictates sustainable and future-proof drug portfolios' for continued attractive returns that biopharmaceutical companies have always provided.

We propose a new radical approach to drug development that is transformative. A core-shell architecture (that represents Cellular Architecture), is based on a multitier architectural design.

\section{Core-Shell architecture (Figure 1)}

In Precision Therapeutic Development, it is a core-shell architecture in which clinical presentation, clinical function processing, and therapeutic drug functions are physically separated. Core-shell architecture provides a model by which drug developers can create flexible and reusable drug clinical candidates. By segregating the precision therapeutic drug into tiers or layers, developers acquire the option of modifying or adding a specific layer, instead of reworking the entire drug formulation. A Core-Shell architecture is typically composed of a clinical presentation tier, a clinical function logic tier, and a therapeutic core tier.

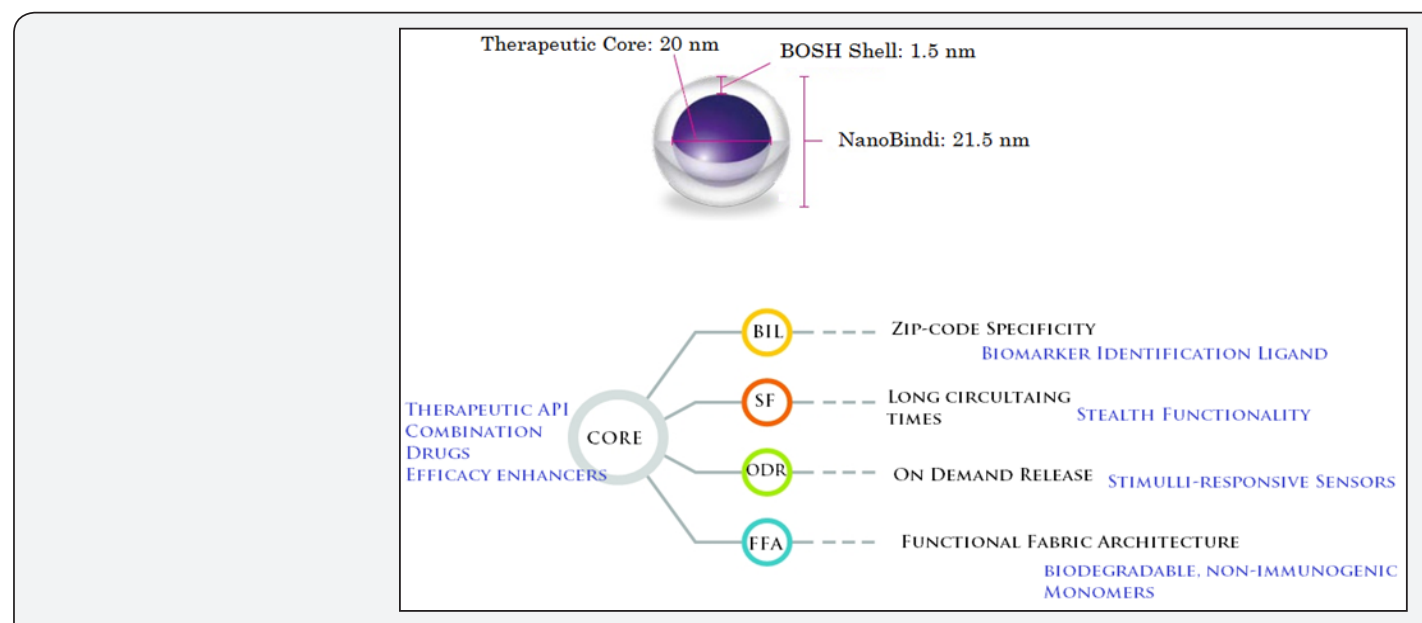

Figure 1: Overview of a Core-Shell precision therapeutic.

While the concepts of layer and tier are often used interchangeably, one fairly common point of view is that there is indeed a difference. This view holds that a layer is a logical structuring mechanism for the elements that make up the functionality solution, while a tier is a physical structuring mechanism for the system infrastructure [4]. For example, a three-layer solution could easily be deployed on a single tier, such as an existing drug [4].

The more usual convention is that the clinical presentation layer (or functional layer) is considered a sublayer of the developmental layer, typically encapsulating the API definition surfacing the supported clinical functionality. The clinical function/business layers can, in fact, be further subdivided to emphasize additional sublayers of distinct functional responsibility. For example, if the alternate route of administration is required, the function presenter sublayer might be used as an additional layer between the clinical interface layer and the clinical function layer (as represented by the model sublayer).

A layer is on top of another, because it depends on it. Every layer can exist without the layers above it, and requires the layers below it to function. Another common view is that layers do not always strictly depend on only on the adjacent layer on below. For example, in a relaxed layered system (as opposed to a strict layered system) a layer can also depend on all the layers below it [4]. 
Core-Shell unified drug development architecture is a precision Therapeutic Development architecture in which the clinical interface (presentation), functional process logic ("clinical outcome rules"), Therapeutic drug core are developed and maintained as independent modules, most often on separate platforms [4].

Apart from the usual advantages of modular developmental platform with well-defined interfaces, the core-shell architecture is intended to allow any of the three tiers to be upgraded or replaced independently in response to changes in requirements or technology. For example, a change of therapeutic drug in the core tier would only affect the core module. Similarly, a new more efficient targeting ligand will only affect a single clinical interface on the functional fabric interface layer.

Typically, the patient presentation layer uses inbuilt clinical function interfaces on the functional fabric that may consist of one or more separate modules encapsulating a therapeutic core that contains the active drug. The middle tier may be multitier itself (in which case the overall architecture is called an "n-tier architecture") in cases of precision therapeutic that needs multiple shells for alternative routes of drug administration.

\section{Three-tier Architecture (Figure 2) \\ Presentation tier}

This is the topmost level of the precision therapeutic. The presentation tier displays information related to clinical functionality such as cell specific targeting, increased circulation for availability, protection from host defense and on demand controlled release. It is integrated on the same plane for the integrated easy access to other tiers by which it puts out the therapeutic drug to the right cells at the right time in the right concentration for optimal clinical-outcome. In simple terms, it is a layer which disease affected cells are presented with the therapeutic drug in a best clinical setting.

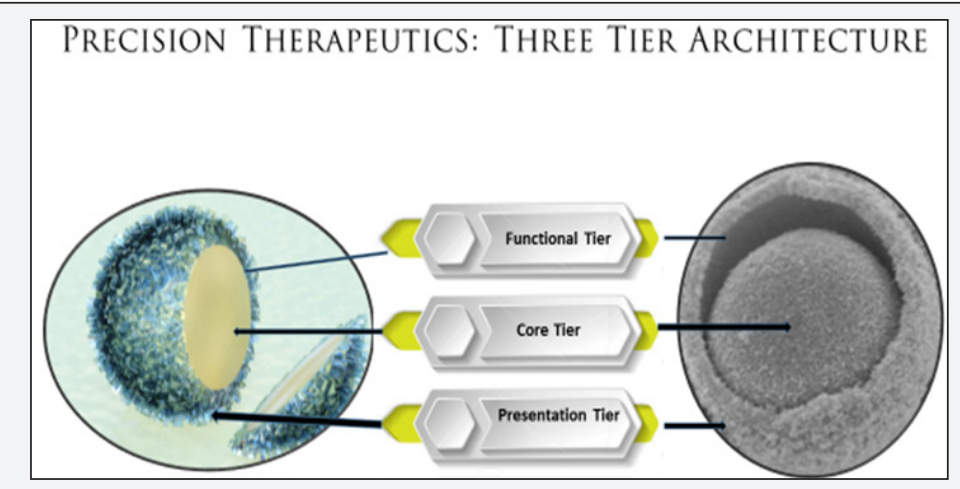

Figure 2 : Precision Therapeutics: Three Tier Architecture.

\section{Infrastructure tier (business functional logic, logic tier, or middle tier)}

The infrastructure or clinical function implementation tier is the functional fabric with integrated clinical interfaces that encapsulates the therapeutic core. The logical tier is pulled out from the presentation tier and, as its own layer, it controls the clinical functionality by performing integration of clinical interfaces on a unified architecture of the functional fabric.

\section{Therapeutic core tier}

The core tier includes the pharmaceutically active ingredient (s), combination products or clinically most relevant pharmaceutical composition. The infrastructure and the presentation tier should provide an API to the targeted cell that is affected by the disease without exposing the drug to other cells or tissues of the patient, or creating side effects in the patient. Avoiding the direct modification to the active drug allows for updates or changes without the presentation or the infrastructure tier being affected by or even aware of the change.
The traditional costs of separation of tiers for implementation and costs to performance in exchange for improved scalability and maintainability are avoided in the precision therapeutics developed on a NanoBindi based drug development platform.

By segregating the precision therapeutic drug design into tiers, drug developers acquire the option of modifying or adding a specific layer, instead of reworking the entire drug development process cycle. A three-tier architecture is typically composed of a clinical functionality tier, a domain logic tier, and a therapeutic drug core tier.

In a logical multilayered architecture for a precision therapeutic system with an clinical outcome-oriented design, the following four are the most common:

a) Presentation layer (a.k.a. clinical interaction layer, view layer, presentation tier in multitier architecture).

b) Clinical Interface layer (a.k.a. service layer or Controller Layer). 
c) Functional layer (a.k.a. Clinical interface logic layer (CILL), domain layer).

d) Core Therapeutic layer.

Apart from the usual advantages of modular drug development processes with well-defined interfaces, the threetier architecture is intended to allow any of the three tiers to be upgraded or replaced independently in response to changes in clinical requirements or drug development process technology. For example, a change of targeting ligand in the presentation tier would only affect the outcome specific clinical interface.

\section{References}

1. Agres T (2011) New Life for Old Drugs. Drug Discovery and Development.

2. DiMasi JA, Grabowski HG, Hansen RW (2016) Innovation in the pharmaceutical industry: New estimates of R\&D costs. J Health Econ 47: 20-33.

3. Herper M The Cost of Creating a New Drug Now $\$ 5$ Billion, Pushing Big Pharma to Change. Forbes.

4. Eckerson WW (1995) Three Tier Client/Server Architecture: Achieving Scalability, Performance, and Efficiency in Client Server Applications. Open Information Systems 3(20): 46-50.

\section{Your next submission with Juniper Publishers will reach you the below assets}

- Quality Editorial service

- Swift Peer Review

- Reprints availability

- E-prints Service

- Manuscript Podcast for convenient understanding

- Global attainment for your research

- Manuscript accessibility in different formats

( Pdf, E-pub, Full Text, Audio)

- Unceasing customer service

Track the below URL for one-step submission https://juniperpublishers.com/online-submission.php 